BULL. AUSTRAL. MATH. SOC.

$58 \mathrm{Al5}, 58 \mathrm{~F} 35,70 \mathrm{Gg}, 70 \mathrm{H35}$

VOL. $25(1982), 309-311$.

\title{
LIE SYMMETRIES OF DIFFERENTIAL EQUATIONS
}

AND DYNAMICAL SYSTEMS

\section{Geoffrey Eamonn Prince}

My primary objective in this work is a detailed exploration of the way in which solutions of ordinary differential equations, especially those of classical mechanics, may be classified and analysed according to their invariance properties under continuous transformation groups. This leads to a group-theoretic treatment of the "symmetry-conservation law" duality in classical physics.

I give the framework of Lie's theory of differential equations from a geometric viewpoint with particular emphasis on the transformation of solutions of the equations under the Lie group which they admit. The theory is then extended to include a new method of construction of first integrals by a study of the solvable structure of certain subalgebras of the invariance algebra. I use this structure to reduce various associated canonical forms of the differential equations to quadratures consequently producing first integrals. In terms of the Newtonian description of dynamical systems this amounts to associating conservation laws with vector fields, that is, symmetries, on $M \times \mathbf{R} \quad(M$ is the configuration space of the system and $R$ is adjoined to deal explicitly with the Newtonian time). An attractive feature of this theory is that, in general, once the Lie algebra is known obtaining the conservation laws is straightforward and the transformation properties of these laws under the group are particularly simple. However, there are two drawbacks; the first is that while all conservation laws are constructible in the majority of cases, situations

Received 7 January 1982. Thesis submitted to La Trobe University, June 1981. Degree approved December 1981. Supervisors: Professor C.J. Eliezer and Dr P.G.L. Leach. 
exist where a system admits no Lie group on $M \times \mathbf{R}$, but where a conservation law is known from other considerations. The second, less important drawback is that even when all conservation laws are obtained there may not be a one-to-one correspondence with the vector fields forming the invariance algebra.

The first part of the thesis finishes with the application of the theory to the Newtonian formulations of the free particle, simple harmonic oscillator and the Kepler problem.

In the second part I deal with conservation laws in Lagrangian mechanics. Using Cartan's geometric approach to Lagrangian systems (see Crampin [1] and Hermann [2]) I look in turn at Cartan's results, Noether's Theorem and its generalizations and what I call the Lie Theory. Cartan's approach of using the evolution space (that is, $T(M) \times \mathbf{R}$ ) as opposed to $M \times \mathrm{R}$ overcomes the two difficulties mentioned earlier quite spectacularly. Cartan introduced the exterior derivative of a certain oneform, now known as the Cartan form, as the fundamental geometric object in Lagrangian mechanics. There is a one-to-one correspondence between the one parameter invariance groups on $T(M) \times \mathbf{R}$ of this object and all the conservation laws of the system, the success being marred only by the fact that it may not be possible to find all conservation laws and their associated symmetries because the generators of one parameter groups on the tangent bundle do not in general form a Lie algebra. I widen the scope of Cartan's idea of invariance and look at the transformation properties of his fundamental geometric object under what Lie might have called the invariance groups of the tangent bundle of the trajectories. In this way, synthesising Lie's insight into invariance and Cartan's geometric intuition, I considerably broaden the current classification of the symmetry - conservation law duality in Lagrangian mechanics (see Sarlet and Cantrijn [3]). The results are illustrated with a treatment of the Lagrangian formulation of the systems dealt with earlier.

\section{References}

[1] M. Crampin, "Constants of the motion in Lagrangian mechanics:", Internat. J. Theoret. Phys. 16 (1977), 741-754. 
[2] Robert Hermann, Differential geometry and the calculus of variations (Mathematics in Science and Engineering, 49. Academic Press, New York and London, 1968).

[3] Willy Sarlet and Frans Cantrijn, "Generalizations of Noether's theorem in classical mechanics", SIAM Rev. 23 (1981), 467-494.

Department of Mathematics,

La Trobe University,

Bundoora,

Victoria 3083,

Australia. 